\title{
Coderivatives of gap function for Minty vector variational inequality
}

Xiaowei Xue 1* $^{\text {and Yu Zhang }}{ }^{2}$

\section{"Correspondence:}

xuexw1@126.com

${ }^{1}$ College of Mathematics and

Statistics, Nanyang Normal

University, Nanyang, Henan 473061,

China

Full list of author information is

available at the end of the article

\begin{abstract}
The purpose of this paper is to investigate coderivatives of the gap function involving the Minty vector variational inequality. First, we discuss the regular coderivative, the normal coderivative, and the mixed coderivative of a class of set-valued maps. Then, by using the relationships between the coderivatives of a set-valued map and its efficient points set-valued map, we obtain the coderivatives of the gap function for the Minty vector variational inequality.
\end{abstract}

MSC: 49K40; 90C29; 90C31; 49J53

Keywords: Minty vector variational inequality; gap function; normal cone; coderivative

\section{Introduction}

The vector variational inequality (for short, $\mathrm{VVI}$ ) and the Minty vector variational inequality (for short, MVVI) have been of great interest in the academic and professional communities ever since the path-breaking paper [1] in the early 1980s. Enormous results on the existence (see $[2,3])$ and stabilities (see $[4,5])$ have been obtained. There are some applications to be found in vector traffic equilibrium problems (see [6, 7]).

It is well known that the concept of gap functions is very important for the study of (VVI) and (MVVI). From the vector optimization point of view, Chen et al. [8] defined the gap function for the (VVI) problem as a set-valued map. Under some suitable coerciveness conditions, Li et al. [9] discussed the differential and sensitivity properties of the set-valued gap functions defined in [8] for (VVI). They also obtained an explicit expression of the contingent derivative for a class of set-valued maps, and some optimality conditions for (VVI) and weak (VVI) by virtue of the gap functions. Later, by the definition of the gap function for Minty vector variational inequalities, some differential and sensitivity results for Minty vector variational inequalities were also obtained in [10]. High-order optimality conditions and differential and sensitivity properties for gap functions of weak (VVI) were also considered (see [11]).

The generalized derivatives mentioned above for set-valued maps are generated by tangent cones to their graphs in primal spaces. Another derivative-like construction for setvalued maps has been introduced by Mordukhovich [12], which is called coderivatives and is generated by normal cones to their graphs in dual spaces. There are numerous applications of coderivatives and the corresponding subdifferential to derive necessary condi-

(c) 2015 Xue and Zhang. This article is distributed under the terms of the Creative Commons Attribution 4.0 International License (http://creativecommons.org/licenses/by/4.0/), which permits unrestricted use, distribution, and reproduction in any medium, provided you give appropriate credit to the original author(s) and the source, provide a link to the Creative Commons license, and indicate if changes were made. 
tions and existence properties in various vector optimization problems, such as [13-15]. Coderivatives have also been applied to sensitivity analysis of scalar (single-objective) optimization problems. We refer the readers to [16-19] for just a few of them.

Recently, Li and Xue [20] discussed the differential and sensitivity properties of the setvalued gap functions defined in [8] for (VVI) via coderivatives. First, they established an explicit expression for computing the normal coderivative and mixed coderivative of a class of set-valued map. Then, through discussing the relations between a set-valued map and its efficient points set-valued map, they investigated sensitivity properties of the gap function for VVI. They also obtained some optimality conditions for (VVI).

Motivated by the work reported in $[10,20]$, in this paper, we make an effort to investigate the coderivatives of Minty vector variational inequality problem in general Banach spaces. First, we establish an explicit expression for computing the regular coderivatives, normal coderivative, and mixed coderivative of a class of set-valued maps. Then, using the relations between coderivatives of a set-valued map and its efficient points set-valued map, we obtain the coderivatives of the gap function for the Minty vector variational inequality. We also give some examples to illustrate the results.

The rest of the paper is organized as follows. In Section 2, we recall the basic definitions and notations from the vector variational inequality, set-valued analysis, and variational analysis. In Section 3, we establish the coderivative of a class of set-valued map. Under some mild conditions, we first give the including relations of the coderivatives of set-valued maps. Then we obtain the explicit expressions under some stronger conditions. In Section 4, we give the coderivatives of the gap function for (MVVI).

Throughout the paper we use the standard notation, with special symbols introduced where they are defined. Unless otherwise stated, all spaces considered are Banach spaces, whose norms are always denoted by $\|\cdot\|$. For any space $X$, we consider its dual space $X^{*}$ equipped with the weak" topology $w^{*}$, where $\langle\cdot, \cdot\rangle$ means the canonical pairing. The closed ball with center $x$ and radius $\eta$ is denoted by $B_{\eta}(x)$. The symbol $A^{*}$ denotes the adjoint operator of a linear continuous operator $A$. If $F: X \rightrightarrows Y$ is a set-valued map, we denote by $\operatorname{dom} F=\{x \in X \mid F(x) \neq \emptyset\}$ and $\operatorname{gph} F=\{(x, y) \in X \times Y \mid y \in F(x)\}$, the domain and graph of $F$, respectively. The notation $\stackrel{*}{\rightarrow}$ stands for weak* convergence in a dual space, while

$x_{n} \stackrel{S}{\rightarrow} x$ means that the sequence $x_{n}$ is contained in the subset $S$ and converges to $x$. For the set-valued map $F: X \rightrightarrows X^{*}$ the expression

$$
\underset{x \rightarrow \bar{x}}{\operatorname{Limsup}} F(x)=\left\{x^{*} \in X^{*} \mid \exists \text { sequences } x_{k} \rightarrow \bar{x}, x_{k}^{*} \stackrel{*}{\rightarrow} x^{*} \text { s.t. } x_{k}^{*} \in F\left(x_{k}\right) \text { for all } k \in \mathbb{N}\right\}
$$

signifies the sequential Painlevé-Kuratowski upper (outer) limit with respect to the norm topology in $X$ and the weak* topology in $X^{*}$. The origins of all real normed spaces are denoted by 0 .

\section{Basic definitions and preliminaries}

Throughout this paper, let $L(X, Y)$ be the set of all linear continuous operators from $X$ to $Y$. For any $A \in L(X, Y)$, we introduce norm

$$
\|A\|_{L}=\sup \{\|A(x)\| \mid\|x\| \leq 1\} .
$$

Since $X, Y$ are Banach spaces, $L(X, Y)$ is also a Banach space with the norm. 
Definition 2.1 Let $F: X \rightarrow L(X, Y)$ be a vector-valued function. $F$ is said to be Fréchet differentiable at $x_{0}$ if and only if there exists a linear continuous operator $\Phi: X \rightarrow L(X, Y)$, such that

$$
\lim _{x \rightarrow x_{0}} \frac{\left\|F(x)-F\left(x_{0}\right)-\Phi\left(x-x_{0}\right)\right\|_{L}}{\left\|x-x_{0}\right\|}=0 .
$$

Obviously, $\Phi$ is unique. We denote derivative $\Phi$ of $F$ at $x_{0}$ by $\nabla F\left(x_{0}\right)$. If, for any $x \in$ $K, F$ is Fréchet differentiable at $x, F$ is said to be Fréchet differentiable on $K$. Therefore, $\nabla F(\cdot): X \rightarrow L(X, Y)$ is a vector-valued function.

In the following of this section, we introduce the basic concepts and constructions of variational analysis and generalized differentiation needed for formulations and justifications of the main results of the paper. Most of the concepts and properties can be found in [21].

Definition 2.2 [22, 23] Let $\Omega \subset X$ be a nonempty subset of a Banach space.

(i) Given $\bar{x} \in \Omega$ and $\varepsilon \geq 0$. The set of $\varepsilon$-normals to $\Omega$ at $\bar{x} \in \Omega$ is defined by

$$
\hat{N}_{\varepsilon}(\bar{x}, \Omega)=\left\{x^{*} \in X^{*} \mid \limsup _{x \rightarrow \bar{x}} \frac{\left\langle x^{*}, x-\bar{x}\right\rangle}{\|x-\bar{x}\|} \leq \varepsilon\right\} .
$$

When $\varepsilon=0$, the set (1) is a cone that is called the regular normal cone (or the prenormal cone) to $\Omega$ at $\bar{x}$ and is denoted by $\hat{N}(\bar{x}, \Omega)$. We put $\hat{N}_{\varepsilon}(\bar{x}, \Omega)=\emptyset$ for all $\varepsilon \geq 0$ if $\bar{x} \notin \Omega$.

(ii) The Mordukhovich normal cone (or basic normal cone) to $\Omega \subset X$ at $\bar{x}$ is defined through the Painlevé-Kuratowski upper (outer) limit as

$$
N(\bar{x}, \Omega)=\operatorname{Limsup}_{x_{k} \rightarrow \bar{x}, \varepsilon_{k} \rightarrow 0_{+}} \hat{N}_{\varepsilon_{k}}\left(x_{k}, \Omega\right) .
$$

Definition 2.3 [12, 24] Consider a set-valued map $\Phi: X \rightrightarrows Y$ between Banach spaces.

(i) The $\varepsilon$-coderivative $\hat{D}_{\varepsilon}^{*} \Phi(\bar{x}, \bar{y})$ at $(\bar{x}, \bar{y})$ is defined through the $\varepsilon$-normal set (1) to the graph as

$$
\hat{D}_{\varepsilon}^{*} \Phi(\bar{x}, \bar{y})\left(y^{*}\right)=\left\{x^{*} \in X^{*} \mid\left(x^{*},-y^{*}\right) \in \hat{N}_{\varepsilon}((\bar{x}, \bar{y}), \operatorname{gph} \Phi)\right\} .
$$

When $\varepsilon=0$, the positive homogeneous set-valued map of $y^{*}$ in (3) is called the regular coderivative of $\Phi$ at $(\bar{x}, \bar{y})$ and denoted by $\hat{D}^{*} \Phi(\bar{x}, \bar{y})(\cdot)$.

(ii) The normal (Mordukhovich) coderivative of $\Phi$ at $(\bar{x}, \bar{y})$ is

$$
D_{N}^{*} \Phi(\bar{x}, \bar{y})\left(y^{*}\right)=\left\{x^{*} \in X^{*} \mid\left(x^{*},-y^{*}\right) \in N((\bar{x}, \bar{y}), \operatorname{gph} \Phi)\right\}
$$

that is, $D_{N}^{*} \Phi(\bar{x}, \bar{y})\left(y^{*}\right)$ is the collection of all $x^{*}$ for which there are sequences $\varepsilon_{k} \rightarrow 0_{+},\left(x_{k}, y_{k}\right) \rightarrow(\bar{x}, \bar{y}),\left(x_{k}^{*}, y_{k}^{*}\right) \stackrel{*}{\rightarrow}\left(x^{*}, y^{*}\right)$ with $\left(x_{k}, y_{k}\right) \in \operatorname{gph} \Phi$ and $x_{k}^{*} \in \hat{D}_{\varepsilon_{k}}^{*} \Phi\left(x_{k}, y_{k}\right)\left(y_{k}^{*}\right)$.

(iii) The mixed coderivative $D_{M}^{*} \Phi(\bar{x}, \bar{y})$ of a set-valued map $\Phi: X \rightrightarrows Y$ at $(\bar{x}, \bar{y})$ is the set-valued map $D_{M}^{*} \Phi(\bar{x}, \bar{y}): Y^{*} \rightrightarrows X^{*}$ defined by

$$
D_{M}^{*} \Phi(\bar{x}, \bar{y})\left(y^{*}\right)=\operatorname{Limsup}_{\left(x_{k}, y_{k}, y_{k}^{*}\right) \rightarrow\left(\bar{x}, \bar{y}, y^{*}\right), \varepsilon_{k} \rightarrow 0_{+}} \hat{D}_{\varepsilon_{k}}^{*} \Phi\left(x_{k}, y_{k}\right)\left(y_{k}^{*}\right),
$$




$$
\begin{aligned}
& \text { i.e., } x^{*} \in D_{M}^{*} \Phi(\bar{x}, \bar{y})\left(y^{*}\right) \text { if and only if there are sequences } \varepsilon_{k} \rightarrow 0_{+} \\
& \left(x_{k}, y_{k}, y_{k}^{*}\right) \rightarrow\left(\bar{x}, \bar{y}, y^{*}\right), x_{k}^{*} \stackrel{*}{\rightarrow} x^{*} \text { with }\left(x_{k}, y_{k}\right) \in \operatorname{gph} \Phi \text {, and } x_{k}^{*} \in \hat{D}_{\varepsilon_{k}}^{*} \Phi\left(x_{k}, y_{k}\right)\left(y_{k}^{*}\right) .
\end{aligned}
$$

It follows from the definitions that $D_{M}^{*} \Phi(\bar{x}, \bar{y})\left(y^{*}\right) \subset D_{N}^{*} \Phi(\bar{x}, \bar{y})\left(y^{*}\right)$ when the equality obviously holds if $Y$ is finite-dimensional. We say that $\Omega$ is regular at $\bar{x} \in \Omega$ if $N(\bar{x}, \Omega)=$ $\hat{N}(\bar{x}, \Omega)$ and $\Phi$ is $N$-regular (resp. $M$-regular) at $(\bar{x}, \bar{y})$ if and only if $D_{N}^{*} \Phi(\bar{x}, \bar{y})=\hat{D}^{*} \Phi(\bar{x}, \bar{y})$ (resp. $D_{M}^{*} \Phi(\bar{x}, \bar{y})=\hat{D}^{*} \Phi(\bar{x}, \bar{y})$ ) (see [24]). The following proposition gives a sufficient condition for the regularity of $\Phi$ and special representations of the coderivatives.

Proposition 2.1 [21] Let $\Phi: X \rightarrow Y$ be Fréchet differentiable at $\bar{x}$. Then

$$
\hat{D}^{*} \Phi(\bar{x})\left(y^{*}\right)=\left\{(\nabla \Phi(\bar{x}))^{*} y^{*}\right\}, \quad \forall y^{*} \in Y^{*}
$$

Moreover, if $\Phi$ is strictly differentiable at $\bar{x}$, i.e., $\Phi$ is single-valued around $\bar{x}$ and

$$
\lim _{x, x^{\prime} \rightarrow \bar{x}}\left[\Phi(x)-\Phi\left(x^{\prime}\right)-\nabla \Phi(\bar{x})\left(x-x^{\prime}\right)\right] /\left\|x-x^{\prime}\right\|=0,
$$

then $\Phi$ is $N$-regular at $\bar{x}$ and one has

$$
D_{N}^{*} \Phi(\bar{x})\left(y^{*}\right)=D_{M}^{*} \Phi(\bar{x})\left(y^{*}\right)=\left\{(\nabla \Phi(\bar{x}))^{*} y^{*}\right\}, \quad \forall y^{*} \in Y^{*} .
$$

We also need some Lipschitzian notions in the following study.

Definition 2.4 [25] Let $f: X \rightarrow Y$ be a single-valued map and $\bar{x} \in \operatorname{dom} f . f$ is said to be local upper Lipschitzian at $\bar{x}$ if there are numbers $\eta>0$ and $L>0$ such that

$$
\|f(x)-f(\bar{x})\| \leq L\|x-\bar{x}\|, \quad \text { for all } x \in B_{\eta}(\bar{x}) \cap \operatorname{dom} f .
$$

We say that a set-valued map $F: X \rightrightarrows Y$ admits a local upper Lipschitzian selection at $(\bar{x}, \bar{y}) \in \operatorname{gph} F$ if there is a single-valued $\operatorname{map} f: \operatorname{dom} F \rightarrow Y$ which is local upper Lipschitzian at $\bar{x}$ satisfying $f(\bar{x})=\bar{y}$ and $f(x) \in F(x)$ for all $x \in \operatorname{dom} F$ in a neighborhood of $\bar{x}$.

Definition 2.5 [26] We say that the domination property holds for the multifunction $F$ : $X \rightrightarrows Y$ around $\bar{x}$ if there exists a neighborhood $U$ of $\bar{x}$ such that

$F(x) \subset \operatorname{Min} F(x)+S, \quad$ for all $x \in U$,

or

$F(x) \subset \operatorname{Max} F(x)-S, \quad$ for all $x \in U$.

\section{Coderivatives of a set-valued map}

In subsequent sections, let $K$ be a closed subset of $X, F: X \rightarrow L(X, Y)$ be a continuous vector-valued map, and

$$
G(x)=\bigcup_{z \in K} F(z)(x-z)
$$


We will discuss the coderivative properties of $G$. First, recall that a set-valued map $H$ : $X \rightrightarrows Y$ is said to be inner semicontinuous at $(\hat{x}, \hat{y}) \in \operatorname{gph} H$ if for every sequence $x_{k} \rightarrow \hat{x}$ with $x_{k} \in \operatorname{dom} H$ there is a sequence $y_{k} \in H\left(x_{k}\right)$ converging to $\hat{y}$ as $k \rightarrow \infty$. $H$ is inner semicompact at $\hat{x}$ if for every sequence $x_{k} \rightarrow \hat{x}$ with $x_{k} \in \operatorname{dom} H$ there is a sequence $y_{k} \in$ $H\left(x_{k}\right)$ that contains a convergent subsequence as $k \rightarrow \infty$.

In the rest of this paper, let $D^{*}$ stand either for the normal coderivative (4) or for the mixed coderivative (5). Since the proof methods of normal coderivative and mixed coderivative are similar, we only show the case of normal coderivative in the following.

Theorem 3.1 Let $\hat{x} \in \operatorname{dom} G, \hat{y} \in G(\hat{x})$, and

$$
M(x, y)=\{z \in K \mid F(z)(x-z)=y\} .
$$

(i) For any $y^{*} \in Y^{*}$,

$$
\hat{D}^{*} G(\hat{x}, \hat{y})\left(y^{*}\right) \subset \bigcap_{\hat{z} \in M(\hat{x}, \hat{y})} F(\hat{z})^{*} y^{*}+\hat{N}(\hat{x}, \operatorname{dom} G) .
$$

(ii) If $M$ is inner semicompact at $(\hat{x}, \hat{y})$, then, for any $y^{*} \in Y^{*}$,

$$
D^{*} G(\hat{x}, \hat{y})\left(y^{*}\right) \subset \bigcup_{\hat{z} \in M(\hat{x}, \hat{y})} F(\hat{z})^{*} y^{*}+N(\hat{x}, \operatorname{dom} G) .
$$

(iii) Given $\hat{z} \in M(\hat{x}, \hat{y})$, if $M$ is inner semicontinuous at $(\hat{x}, \hat{y}, \hat{z})$, then, for any $y^{*} \in Y^{*}$,

$$
D^{*} G(\hat{x}, \hat{y})\left(y^{*}\right) \subset F(\hat{z})^{*} y^{*}+N(\hat{x}, \operatorname{dom} G)
$$

Proof (i) For any $x^{*} \in \hat{D}^{*} G(\hat{x}, \hat{y})\left(y^{*}\right)$, by the definitions of regular coderivative and regular normal cone,

$$
\limsup _{\left(x_{k}, y_{k}\right) \stackrel{\operatorname{ghp} G}{\rightarrow}(\hat{x}, \hat{y})} \frac{\left\langle\left(x^{*},-y^{*}\right),\left(x_{k}-\hat{x}, y_{k}-\hat{y}\right)\right\rangle}{\left\|x_{k}-\hat{x}\right\|+\left\|y_{k}-\hat{y}\right\|} \leq 0 .
$$

Then, for any $z_{k} \in K$ satisfying $z_{k} \rightarrow \hat{z}$, we have

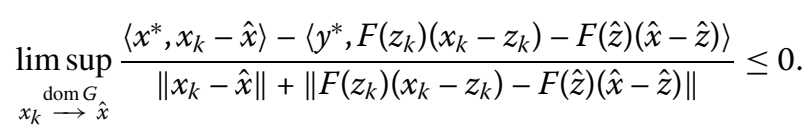

Especially, let $z_{k}=\hat{z}$, we have

$$
\underset{\substack{\operatorname{dom} G \\ x_{k} \stackrel{x}{\longrightarrow}}}{\limsup } \frac{\left\langle x^{*}, x_{k}-\hat{x}\right\rangle-\left\langle y^{*}, F(\hat{z})\left(x_{k}-\hat{x}\right)\right\rangle}{\left\|x_{k}-\hat{x}\right\|+\left\|F(\hat{z})\left(x_{k}-\hat{x}\right)\right\|} \leq 0 .
$$

Thus,

$$
\limsup _{\substack{\operatorname{dom} G \\ x_{k} \stackrel{x}{\longrightarrow}}} \frac{\left\langle x^{*}, x_{k}-\hat{x}\right\rangle-\left\langle F(\hat{z})^{*} y^{*}, x_{k}-\hat{x}\right\rangle}{\left\|x_{k}-\hat{x}\right\|} \leq 0 .
$$


This ensures that $x^{*}-F(\hat{z})^{*} y^{*} \in \hat{N}(\hat{x}, \operatorname{dom} G)$. So we have

$$
\hat{D}^{*} G(\hat{x}, \hat{y})\left(y^{*}\right) \subset F(\hat{z})^{*} y^{*}+\hat{N}(\hat{x}, \operatorname{dom} G) .
$$

(ii) For any $x^{*} \in D^{*} G(\hat{x}, \hat{y})\left(y^{*}\right)$, by the definitions of coderivatives and normal cone, there are sequences of vector $\left(x_{k}, y_{k}\right) \rightarrow(\hat{x}, \hat{y}),\left(x_{k}^{*}, y_{k}^{*}\right) \stackrel{*}{\rightarrow}\left(x^{*}, y^{*}\right)\left(y_{k}^{*} \rightarrow y^{*}\right.$ for the case of a mixed coderivative) and $\varepsilon_{k} \downarrow 0$ such that $y_{k} \in G\left(x_{k}\right)$ and

$$
\limsup _{\left(x_{k_{i}}, y_{k_{i}}\right) \stackrel{\operatorname{gph} G}{\rightarrow}\left(x_{k}, y_{k}\right)} \frac{\left\langle\left(x_{k}^{*},-y_{k}^{*}\right),\left(x_{k_{i}}-x_{k}, y_{k_{i}}-y_{k}\right)\right\rangle}{\left\|x_{k_{i}}-x_{k}\right\|+\left\|y_{k_{i}}-y_{k}\right\|} \leq \varepsilon_{k} .
$$

Since $M$ is inner semicompact at $(\hat{x}, \hat{y})$, for the above $\left(x_{k}, y_{k}\right)$, there exists a sequence of $z_{k} \in M\left(x_{k}, y_{k}\right)$ that contains a subsequence converging to some $\hat{z}$. Since $K$ is closed, we have $\hat{z} \in K$. Let $k \rightarrow \infty$ in $y_{k}=F\left(z_{k}\right)\left(x_{k}-z_{k}\right)$, we have $\hat{y}=F(\hat{z})(\hat{x}-\hat{z})$, which implies $\hat{z} \in M(\hat{x}, \hat{y})$. Then, for any $z_{k_{i}} \in K$ satisfying $z_{k_{i}} \rightarrow z_{k}$, we have

$$
\underset{\substack{\operatorname{dom} G \\ x_{k_{i}} \rightarrow x_{k}}}{\limsup } \frac{\left\langle x_{k}^{*}, x_{k_{i}}-x_{k}\right\rangle-\left\langle y_{k}^{*}, F\left(z_{k_{i}}\right)\left(x_{k_{i}}-z_{k_{i}}\right)-F\left(z_{k}\right)\left(x_{k}-z_{k}\right)\right\rangle}{\left\|x_{k_{i}}-x_{k}\right\|+\left\|F\left(z_{k_{i}}\right)\left(x_{k_{i}}-z_{k_{i}}\right)-F\left(z_{k}\right)\left(x_{k}-z_{k}\right)\right\|} \leq \varepsilon_{k} .
$$

Especially, let $z_{k_{i}}=z_{k}$, we have

$$
\underset{\substack{\operatorname{dom} G \\ x_{k_{i}} \rightarrow x_{k}}}{\limsup } \frac{\left\langle x_{k}^{*}, x_{k_{i}}-x_{k}\right\rangle-\left\langle y_{k}^{*}, F\left(z_{k}\right)\left(x_{k_{i}}-x_{k}\right)\right\rangle}{\left\|x_{k_{i}}-x_{k}\right\|+\left\|F\left(z_{k}\right)\left(x_{k_{i}}-x_{k}\right)\right\|} \leq \varepsilon_{k} .
$$

Thus,

$$
\underset{\substack{\operatorname{dom} G \\ x_{k_{i}} \rightarrow x_{k}}}{\limsup } \frac{\left\langle x_{k}^{*}, x_{k_{i}}-x_{k}\right\rangle-\left\langle F\left(z_{k}\right)^{*} y_{k}^{*},\left(x_{k_{i}}-x_{k}\right)\right\rangle}{\left\|x_{k_{i}}-x_{k}\right\|} \leq \varepsilon_{k}^{\prime},
$$

where $\varepsilon_{k}^{\prime}=\left(1+\left\|F\left(z_{k}\right)\right\|\right) \varepsilon_{k} \rightarrow 0$ as $k \rightarrow \infty$. This ensures that $x^{*}-F(\hat{z})^{*} y^{*} \in N(\hat{x}, \operatorname{dom} G)$. So we have

$$
D^{*} G(\hat{x}, \hat{y})\left(y^{*}\right) \subset \bigcup_{\hat{z} \in M(\hat{x}, \hat{y})} F(\hat{z})^{*} y^{*}+N(\hat{x}, \operatorname{dom} G)
$$

(iii) It can be proved similarly to the case (ii), since for any sequence $\left(x_{k}, y_{k}\right) \rightarrow(\hat{x}, \hat{y})$, by the inner semicontinuous assumption of $M$, there exists a sequence $z_{k} \in M\left(x_{k}, y_{k}\right)$ converging to $\hat{z}$. This complete the proof.

Now we turn to the converse inclusion.

Theorem 3.2 Let $\hat{x} \in \operatorname{dom} G, \hat{y} \in G(\hat{x})$, and $\hat{z} \in M(\hat{x}, \hat{y})$. If $F$ is local upper Lipschitzian at $\hat{z}$, and $M$ admits a local upper Lipschitzian selection at $(\hat{x}, \hat{y}, \hat{z})$, then, for any $y^{*} \in Y^{*}$ satisfying $F(\hat{z})^{*} y^{*} \in \hat{D}^{*} F(\hat{z})\left(y^{*}(\hat{x}-\hat{z})^{*}\right)$,

$$
F(\hat{z})^{*} y^{*}+\hat{N}(\hat{x}, \operatorname{dom} G) \subset \hat{D}^{*} G(\hat{x}, \hat{y})\left(y^{*}\right) .
$$


Moreover, if $\operatorname{dom} G$ is regular at $\hat{x}$, then, for any $y^{*} \in Y^{*}$ satisfying $F(\hat{z})^{*} y^{*} \in \hat{D}^{*} F(\hat{z})\left(y^{*}(\hat{x}-\right.$ $\left.\hat{z})^{*}\right)$,

$$
F(\hat{z})^{*} y^{*}+N(\hat{x}, \operatorname{dom} G) \subset D^{*} G(\hat{x}, \hat{y})\left(y^{*}\right) .
$$

Proof Let $F(\hat{z})^{*} y^{*} \in \hat{D}^{*} F(\hat{z})\left(y^{*}(\hat{x}-\hat{z})^{*}\right)$ and $x^{*} \in \hat{N}(\hat{x}$, dom $G)$. Then we have

$$
\underset{z \rightarrow \hat{z}}{\limsup } \frac{\left\langle F(\hat{z})^{*} y^{*}, z-\hat{z}\right\rangle-\left\langle y^{*}(\hat{x}-\hat{z})^{*}, F(z)-F(\hat{z})\right\rangle}{\|z-\hat{z}\|+\|F(z)-F(\hat{z})\|_{L}} \leq 0
$$

and

$$
\underset{x \rightarrow \operatorname{dom}_{\hat{x}}}{\limsup } \frac{\left\langle x^{*}, x-\hat{x}\right\rangle}{\|x-\hat{x}\|} \leq 0 .
$$

This means that, for any $\varepsilon>0$, one can find $\eta_{1}>0, \eta_{2}>0$ such that

$$
\left\langle y^{*}, F(\hat{z})(z-\hat{z})\right\rangle-\left\langle y^{*},(F(z)-F(\hat{z}))(\hat{x}-\hat{z})\right\rangle \leq \varepsilon\left(\|z-\hat{z}\|+\|F(z)-F(\hat{z})\|_{L}\right)
$$

and

$$
\left\langle x^{*}, x-\hat{x}\right\rangle \leq \varepsilon\|x-\hat{x}\|
$$

for all $z \in B_{\eta_{1}}(\hat{z}), x \in B_{\eta_{2}}(\hat{x})$.

Since $M$ admits a local upper Lipschitzian selection at $(\hat{x}, \hat{y}, \hat{z})$, for any $(x, y) \stackrel{\operatorname{gph} G}{\longrightarrow}(\hat{x}, \hat{y})$, there are a constant $t>0$ and $z \in M(x, y)$ such that $\|z-\hat{z}\| \leq t(\|x-\hat{x}\|+\|y-\hat{y}\|)$. Furthermore, the locally upper Lipschitzian assumption of $F$ ensures that $\|F(z)-F(\hat{z})\|_{L} \leq L\|z-\hat{z}\|$ for some $L>0$ whenever $z \rightarrow \hat{z}$. Thus, for any $(x, y) \stackrel{\operatorname{gph} G}{\longrightarrow}(\hat{x}, \hat{y})$, we have the inequalities

$$
\begin{aligned}
\left\langle x^{*}+\right. & \left.F(\hat{z})^{*} y^{*}, x-\hat{x}\right\rangle-\left\langle y^{*}, y-\hat{y}\right\rangle \\
= & \left\langle x^{*}, x-\hat{x}\right\rangle+\left\langle F(\hat{z})^{*} y^{*}, x-\hat{x}\right\rangle-\left\langle y^{*}, F(z)(x-z)-F(\hat{z})(\hat{x}-\hat{z})\right\rangle \\
= & \left\langle x^{*}, x-\hat{x}\right\rangle+\left\langle y^{*}, F(\hat{z})(z-\hat{z})\right\rangle-\left\langle y^{*},(F(z)-F(\hat{z}))(\hat{x}-\hat{z})\right\rangle \\
& -\left\langle y^{*},(F(z)-F(\hat{z}))((x-\hat{x})-(z-\hat{z}))\right\rangle \\
\leq & \varepsilon(\|x-\hat{x}\|)+\varepsilon(\|z-\hat{z}\|+\|F(z)-F(\hat{z})\|)-\left\langle y^{*},(F(z)-F(\hat{z}))((x-\hat{x})-(z-\hat{z}))\right\rangle \\
\leq & \varepsilon(\|x-\hat{x}\|)+\varepsilon(\|z-\hat{z}\|+L\|z-\hat{z}\|)+L\|z-\hat{z}\|\left\|y^{*}\right\|\|(x-\hat{x})-(z-\hat{z})\| \\
\leq & (L t+t+1) \varepsilon(\|x-\hat{x}\|+\|y-\hat{y}\|)+L(t+1)\|z-\hat{z}\|\left\|y^{*}\right\|(\|x-\hat{x}\|+\|y-\hat{y}\|) \\
= & \left((L t+t+1) \varepsilon+L t(t+1)\left\|y^{*}\right\|(\|x-\hat{x}\|+\|y-\hat{y}\|)\right)(\|x-\hat{x}\|+\|y-\hat{y}\|) .
\end{aligned}
$$

Since $\varepsilon>0$ is chosen arbitrarily, we have

$$
\limsup _{(x, y) \stackrel{\operatorname{gph} G}{\longrightarrow}(\hat{x}, \hat{y})} \frac{\left\langle x^{*}+F(\hat{z})^{*} y^{*}, x-\hat{x}\right\rangle-\left\langle y^{*}, y-\hat{y}\right\rangle}{\|x-\hat{x}\|+\|y-\hat{y}\|} \leq 0 .
$$


By the definition of regular coderivative, $x^{*}+F(\hat{z})^{*} y^{*} \in \hat{D}^{*} G(\hat{x}, \hat{y})\left(y^{*}\right)$, and then

$$
F(\hat{z})^{*} y^{*}+\hat{N}(\hat{x}, \operatorname{dom} G) \subset \hat{D}^{*} G(\hat{x}, \hat{y})\left(y^{*}\right) .
$$

Moreover, if $K$ is regular at $\hat{x}$, then $N(\hat{x}, \operatorname{dom} G)=\hat{N}(\hat{x}, \operatorname{dom} G)$ and the residual part is obvious.

Remark 3.1 In fact, since the local upper Lipschitzian selection of $M$ implies the inner semicontinuity and inner semicompactness of $M$, the converse include relations in Theorem 3.2 can be written as equalities.

Corollary 3.1 Let $\hat{z} \in M(\hat{x}, \hat{y})$. If $F$ is Fréchet differentiable at $\hat{z}$, and $M$ admits a local upper Lipschitzian selection at $(\hat{x}, \hat{y}, \hat{z})$, then, for any $y^{*} \in Y^{*}$ satisfying $F(\hat{z})^{*} y^{*}=\nabla F(\hat{z})^{*}\left(y^{*}(\hat{x}-\right.$ $\left.\hat{z})^{*}\right)$, we have

$$
\hat{D}^{*} G(\hat{x}, \hat{y})\left(y^{*}\right)=F(\hat{z})^{*} y^{*}+\hat{N}(\hat{x}, \operatorname{dom} G) .
$$

Moreover, if $\operatorname{dom} G$ is regular at $\hat{x}$, then $G$ is $N$-regular at $(\hat{x}, \hat{y})$ and for any $y^{*} \in Y^{*}$ satisfying $F(\hat{z})^{*} y^{*}=\nabla F(\hat{z})^{*}\left(y^{*}(\hat{x}-\hat{z})^{*}\right)$, we have

$$
D^{*} G(\hat{x}, \hat{y})\left(y^{*}\right)=F(\hat{z})^{*} y^{*}+N(\hat{x}, \operatorname{dom} G) .
$$

Proof Since $F$ is Fréchet differentiable at $\hat{z}$, then $F$ is locally upper Lipschitzian at $\hat{x}$, and for any $y^{*} \in Y^{*}, \hat{D}^{*} F(\hat{z})\left(y^{*}(\hat{x}-\hat{z})^{*}\right)=\nabla F(\hat{z})^{*}\left(y^{*}(\hat{x}-\hat{z})^{*}\right)$. The first equality relation immediately follows from Theorems 3.1, 3.2.

Now assume that $\operatorname{dom} G$ is regular at $\hat{x}$, we prove the second part of the corollary. Obviously, the upper Lipschitz selection property of $M$ implies that $M$ is inner semicontinuous at $(\hat{x}, \hat{y}, \hat{z})$. Thus, for any $y^{*} \in Y^{*}$ satisfying $F(\hat{z})^{*} y^{*}=\nabla F(\hat{z})^{*}\left(y^{*}(\hat{x}-\hat{z})^{*}\right)$, we have

$$
\begin{aligned}
D^{*} G(\hat{x}, \hat{y})\left(y^{*}\right) & \subset F(\hat{z})^{*} y^{*}+N(\hat{x}, \operatorname{dom} G)=F(\hat{z})^{*} y^{*}+\hat{N}(\hat{x}, \operatorname{dom} G) \\
& \subset \hat{D}^{*} G(\hat{x}, \hat{y})\left(y^{*}\right) \subset D^{*} G(\hat{x}, \hat{y})\left(y^{*}\right)
\end{aligned}
$$

The proof is completed.

We give an example to illustrate Theorems 3.1 and 3.2.

Example 3.1 Let $X=Y=\mathbb{R}, K=[-1,1], S=\mathbb{R}_{+}$, and $F(x)=x^{2}$. Let $\hat{x}=-1, \hat{y}=0$. Then we have

$$
G(x)= \begin{cases}{[0, x+1],} & x>1 \\ {[x-1, x+1],} & -1 \leq x \leq 1 \\ {[x-1,0],} & x \leq-1\end{cases}
$$

By direct computing, we have $M(\hat{x}, \hat{y})=\{-1,0\}, \hat{N}(\hat{x}, \operatorname{dom} G)=N(\hat{x}, \operatorname{dom} G)=\{0\}$,

$$
\hat{D}^{*} G(\hat{x}, \hat{y})\left(y^{*}\right)= \begin{cases}0, & y^{*}=0 \\ \emptyset, & \text { else }\end{cases}
$$


and

$$
D^{*} G(\hat{x}, \hat{y})\left(y^{*}\right)= \begin{cases}\left\{y^{*}, 0\right\}, & y^{*} \leq 0 \\ \emptyset, & \text { else }\end{cases}
$$

Then, for any $y^{*} \in \mathbb{R}$, we have

$$
\hat{D}^{*} G(\hat{x}, \hat{y})\left(y^{*}\right) \subset \bigcap_{\hat{z} \in M(\hat{x}, \hat{y})} F(\hat{z})^{*} y^{*}+\hat{N}(\hat{x}, \operatorname{dom} G)
$$

and

$$
D^{*} G(\hat{x}, \hat{y})\left(y^{*}\right) \subset \bigcup_{\hat{z} \in M(\hat{x}, \hat{y})} F(\hat{z})^{*} y^{*}+N(\hat{x}, \operatorname{dom} G) .
$$

However, for any $\hat{z} \in M(\hat{x}, \hat{y})$, the inclusion

$$
D^{*} G(\hat{x}, \hat{y})\left(y^{*}\right) \subset F(\hat{z})^{*} y^{*}+N(\hat{x}, \operatorname{dom} G)
$$

does not hold. This is so because $M$ is not inner semicontinuous at any $(\hat{x}, \hat{y}, \hat{z})$. For example, let $(\hat{x}, \hat{y}, \hat{z})=(-1,0,-1)$, we can find a sequence $\left(x_{k}, y_{k}\right)=\left(-1-\frac{1}{k}, 0\right)$ which does not have a sequence $z_{k} \in M\left(x_{k}, y_{k}\right)$ converging to $\hat{z}$ as $k \rightarrow \infty$.

On the other hand, since $M$ does not admit a local upper Lipschitzian selection at $(\hat{x}, \hat{y}, \hat{z})=(-1,0,-1)$, the converse inclusions

$$
F(\hat{z})^{*} y^{*}+\hat{N}(\hat{x}, \operatorname{dom} G) \subset \hat{D}^{*} G(\hat{x}, \hat{y})\left(y^{*}\right)
$$

and

$$
F(\hat{z})^{*} y^{*}+N(\hat{x}, \operatorname{dom} G) \subset D^{*} G(\hat{x}, \hat{y})\left(y^{*}\right)
$$

do not hold for any $y^{*} \in \mathbb{R}$ though $F$ is continuous differential at $\hat{z}$ and dom $G$ is regular at $\hat{x}$.

\section{Coderivatives of gap functions}

Let $X, Y$ be Banach spaces and $S \subset Y$ be a closed convex and point cone. Given a nonempty closed set $K \subset X$ and a map $F: K \rightarrow L(X, Y)$, where $L(X, Y)$ is a set of all linear continuous operators from $X$ to $Y$, the Minty vector variational inequality is to find $x^{*} \in K$ such that

$$
F(x)\left(x-x^{*}\right) \notin-S \backslash\left\{0_{Y}\right\}, \quad \forall x \in K
$$

Definition 4.1 Let $S$ be a closed convex and pointed cone in $Y$ with nonempty interior. A set-valued map $N: X \rightrightarrows Y$ is said to be a gap function of (MVVI) if and only if

(a) $0 \in N(\hat{x})$ if and only if $\hat{x}$ solves (MVVI);

(b) $N(x) \cap(-S \backslash\{0\})=\emptyset, \forall x \in K$. 
Consider set-valued map $N: X \rightrightarrows Y$ defined by

$$
N(x):=\operatorname{Max}_{S} G(x)=\operatorname{Max}_{S} \bigcup_{z \in K} F(z)(x-z), \quad x \in K,
$$

where the symbol $\operatorname{Max}_{S}$ denotes the collection of efficient points. That is, for a set $A \in Y$,

$$
\operatorname{Max}_{S} A:=\left\{a \in A \mid \nexists a^{\prime} \in A \text { s.t. } a^{\prime}-a \in S \backslash\left\{0_{Y}\right\}\right\} .
$$

By Theorem 4.2 in [10], $N$ is a gap function of (MVVI). So, (MVVI) is equivalent to the following set-valued optimization problem:

$\operatorname{Min}_{S} N(x)$ subject to $x \in K$.

In this section, we discuss the coderivative $D^{*} N$.

Theorem 4.1 Let $K$ be a compact set, $\hat{x} \in K, \hat{y} \in N(\hat{x})$, and

$$
M(x, y)=\{z \in K \mid F(z)(x-z)=y\} .
$$

(i) For any $y^{*} \in Y^{*}$ satisfying $\sup _{s \in S \backslash\{0\}} \frac{\left|y^{*}, s\right\rangle}{\|s\|}=: v<0$,

$$
\hat{D}^{*} N(\hat{x}, \hat{y})\left(y^{*}\right) \subset \bigcap_{\hat{z} \in M(\hat{x}, \hat{y})} F(\hat{z})^{*} y^{*}+\hat{N}(\hat{x}, K)
$$

(ii) If $M$ is inner semicompact at $(\hat{x}, \hat{y})$, then, for any $y^{*} \in Y^{*}$ satisfying

$$
\begin{aligned}
\sup _{s \in S \backslash\{0\}} \frac{\left|y^{*}, s\right\rangle}{\|s\|}=: v<0, \\
D_{M}^{*} N(\hat{x}, \hat{y})\left(y^{*}\right) \subset \bigcup_{\hat{z} \in M(\hat{x}, \hat{y})} F(\hat{z})^{*} y^{*}+N(\hat{x}, K) .
\end{aligned}
$$

(iii) Given $\hat{z} \in M(\hat{x}, \hat{y})$, if $M$ is inner semicontinuous at $(\hat{x}, \hat{y}, \hat{z})$, then, for any $y^{*} \in Y^{*}$ satisfying $\sup _{s \in S \backslash\{0\}} \frac{\left\langle y^{*}, s\right\rangle}{\|s\|}=: v<0$,

$$
D_{M}^{*} N(\hat{x}, \hat{y})\left(y^{*}\right) \subset F(\hat{z})^{*} y^{*}+N(\hat{x}, K) .
$$

In addition to the assumption of case (ii) and (iii), respectively, if $K$ has a compact base, then the including relations in case (ii) and (iii) hold for normal coderivatives.

Proof (i) First, we show that $G$ is compact at any $x \in K$, which ensures that $G$ is locally compact around $\hat{x}$. Give a sequence $\left\{\left(x_{i}, y_{i}\right)\right\} \subset \operatorname{gph} G$ satisfying $x_{i} \rightarrow x$. By the construction of $G$, there exist $z_{i} \in K$ such that $y_{i}=F\left(z_{i}\right)\left(x-z_{i}\right)$. Since $K$ is a compact set, we assume without loss of generality that $z_{i} \rightarrow z \in K$. The continuity of $F$ implies that $y_{i}=F\left(z_{i}\right)(x-$ $\left.z_{i}\right) \rightarrow F(z)(x-z):=y \in G(x)$. Therefore, $G$ is compact at any $x \in K$ and then $G(x)$ is a compact set for any $x \in K$.

Since $G$ is compact at any $x \in K, G(x)$ is a compact set for any $x \in K$. So the domination property for $-G$ holds around $\hat{x}$. That is, $-G(x) \subset \operatorname{Min}_{S}(-G(x))+S$ for any $x \in K$. Therefore, 
we have $-G(x)+S=\operatorname{Min}_{S}(-G(x))+S$, which implies $-G(x)+S=-N(x)+S$. Thus, for any $y^{*} \in Y^{*}$, by the definitions of regular coderivatives, we have

$$
\hat{D}^{*}(-G+S)(\hat{x},-\hat{y})\left(-y^{*}\right)=\hat{D}^{*}(-N+S)(\hat{x},-\hat{y})\left(-y^{*}\right) .
$$

Obviously $-G$ is also compact at any $x \in K$. According to [20], Proposition 4.3, this implies that $-N$ is locally order semicontinuous around $(\hat{x},-\hat{y})$ and then $-G$ is order semicontinuous at $(\hat{x},-\hat{y})$. Employing [20], Proposition 4.2, we have, for any $y^{*} \in Y^{*}$ satisfying $\sup _{s \in S \backslash\{0\}} \frac{\left\langle y^{*}, s\right\rangle}{\|s\|}=: v<0$,

$$
\begin{aligned}
\hat{D}^{*} N(\hat{x}, \hat{y})\left(y^{*}\right) & =\hat{D}^{*}(-N)(\hat{x},-\hat{y})\left(-y^{*}\right) \\
& =\hat{D}^{*}(-N+S)(\hat{x},-\hat{y})\left(-y^{*}\right) \\
& =\hat{D}^{*}(-G+S)(\hat{x},-\hat{y})\left(-y^{*}\right) \\
& =\hat{D}^{*}(-G)(\hat{x},-\hat{y})\left(-y^{*}\right) \\
& =\hat{D}^{*} G(\hat{x}, \hat{y})\left(y^{*}\right) .
\end{aligned}
$$

Note that $\operatorname{dom} G=K$, the inclusions follow from Theorem 3.1.

(ii) Using [20], Proposition 4.1, we get, for any $y^{*} \in Y^{*}$ satisfying $\sup _{s \in S \backslash\{0\}} \frac{\left\langle y^{*}, s\right\rangle}{\|s\|}=: v<0$,

$$
D_{M}^{*}(-N)(\hat{x},-\hat{y})\left(-y^{*}\right)=D_{M}^{*}(-N+S)(\hat{x},-\hat{y})\left(-y^{*}\right)
$$

and

$$
D_{M}^{*}(-G+S)(\hat{x},-\hat{y})\left(-y^{*}\right) \subset D_{M}^{*}(-G)(\hat{x},-\hat{y})\left(-y^{*}\right) .
$$

Thus, case (ii) and (iii) can be proved similar to case (i).

Moreover, if for $K$ we have the compact case, then according to [27], Proposition 3.5, for any $y^{*} \in Y^{*}$ satisfying $\sup _{s \in S \backslash\{0\}} \frac{\left\langle v^{*}, s\right\rangle}{\|s\|}=: v<0$, we have

$$
D_{N}^{*}(-N)(\hat{x},-\hat{y})\left(-y^{*}\right)=D_{N}^{*}(-N+S)(\hat{x},-\hat{y})\left(-y^{*}\right)
$$

and

$$
D_{N}^{*}(-G+S)(\hat{x},-\hat{y})\left(-y^{*}\right) \subset D_{N}^{*}(-G)(\hat{x},-\hat{y})\left(-y^{*}\right) .
$$

Thus, the including relations in case (ii) and (iii) hold for normal coderivatives. This completes the proof.

Example 4.1 Let $X, Y, K, F,(\hat{x}, \hat{y})$ as in Example 3.1. Obviously

$$
N(x)= \begin{cases}x+1, & x \in K \\ \emptyset, & \text { else. }\end{cases}
$$

By direct computing, we have $\hat{N}(\hat{x}, K)=N(\hat{x}, K)=\mathbb{R}_{-}$and

$$
\hat{D}^{*} N(\hat{x}, \hat{y})\left(y^{*}\right)=D^{*} N(\hat{x}, \hat{y})\left(y^{*}\right)=\left\{x^{*} \in \mathbb{R} \mid x^{*} \leq y^{*}\right\} .
$$


Then, for any $y^{*} \in Y^{*}$ satisfying $\sup _{s \in S \backslash\{0\}} \frac{\left\langle\psi^{*}, s\right\rangle}{\|s\|}=: v<0$, i.e., $y^{*}<0$,

$$
\hat{D}^{*} N(\hat{x}, \hat{y})\left(y^{*}\right) \subset \bigcap_{\hat{z} \in M(\hat{x}, \hat{y})} F(\hat{z})^{*} y^{*}+\hat{N}(\hat{x}, K)
$$

and

$$
D^{*} N(\hat{x}, \hat{y})\left(y^{*}\right) \subset \bigcup_{\hat{z} \in M(\hat{x}, \hat{y})} F(\hat{z})^{*} y^{*}+N(\hat{x}, K)
$$

Theorem 4.2 Let $K$ be a compact set, $\hat{x} \in K, \hat{y} \in N(\hat{x})$, and $\hat{z} \in M(\hat{x}, \hat{y})$.

(i) If $F$ is local upper Lipschitzian relative to $K$ at $\hat{z}$, and $M$ admits a local upper Lipschitzian selection at $(\hat{x}, \hat{y}, \hat{z})$, then, for any $y^{*} \in Y^{*}$ satisfying $F(\hat{z})^{*} y^{*} \in \hat{D}^{*} F(\hat{z})\left(y^{*}(\hat{x}-\hat{z})^{*}\right)$ and $\sup _{s \in S \backslash\{0\}} \frac{\left\langle y^{*}, s\right\rangle}{\|s\|}=: v<0$,

$$
F(\hat{z})^{*} y^{*}+\hat{N}(\hat{x}, K) \subset \hat{D}^{*} N(\hat{x}, \hat{y})\left(y^{*}\right)
$$

(ii) In addition to the conditions in (i), if $K$ is regular at $\hat{x}$, then, for any $y^{*} \in Y^{*}$ satisfying $F(\hat{z})^{*} y^{*} \in \hat{D}^{*} F(\hat{z})\left(y^{*}(\hat{x}-\hat{z})^{*}\right)$ and $\sup _{s \in S \backslash\{0\}} \frac{\left\langle y^{*}, s\right\rangle}{\|s\|}=: v<0$,

$$
F(\hat{z})^{*} y^{*}+N(\hat{x}, K) \subset D^{*} N(\hat{x}, \hat{y})\left(y^{*}\right)
$$

Proof It immediately follows from Theorem 3.2 and the proof of Theorem 4.1.

Similarly to Corollary 3.1 we have the following result.

Corollary 4.1 Let $K$ be a compact set, $\hat{x} \in K, \hat{y} \in N(\hat{x})$, and $\hat{z} \in M(\hat{x}, \hat{y})$.

(i) If $F$ is Fréchet differentiable at $\hat{z}$, and $M$ admits a local upper Lipschitzian selection at $(\hat{x}, \hat{y}, \hat{z})$, then, for any $y^{*} \in Y^{*}$ satisfying $F(\hat{z})^{*} y^{*}=\nabla F(\hat{z})^{*}\left(y^{*}(\hat{x}-\hat{z})^{*}\right)$ and $\sup _{s \in S \backslash\{0\}} \frac{\left\langle y^{*}, s\right\rangle}{\|s\|}=: v<0$, we have

$$
\hat{D}^{*} N(\hat{x}, \hat{y})\left(y^{*}\right)=F(\hat{z})^{*} y^{*}+\hat{N}(\hat{x}, K)
$$

(ii) In addition to the conditions in case (i), if $K$ is regular at $\hat{x}$, then $N$ is $M$-regular at $(\hat{x}, \hat{y})$, and for any $y^{*} \in Y^{*}$ satisfying $F(\hat{z})^{*} y^{*}=\nabla F(\hat{z})^{*}\left(y^{*}(\hat{x}-\hat{z})^{*}\right)$ and $\sup _{s \in S \backslash\{0\}} \frac{\left\langle v^{*}, s\right\rangle}{\|s\|}=: v<0$, we have

$$
D_{M}^{*} N(\hat{x}, \hat{y})\left(y^{*}\right)=F(\hat{z})^{*} y^{*}+N(\hat{x}, K)
$$

(iii) In addition to the conditions in case (ii), if $K$ has a compact base, then $N$ is $N$-regular at $(\hat{x}, \hat{y})$, and for any $y^{*} \in Y^{*}$ satisfying $F(\hat{z})^{*} y^{*}=\nabla F(\hat{z})^{*}\left(y^{*}(\hat{x}-\hat{z})^{*}\right)$ and $\sup _{s \in S \backslash\{0\}} \frac{\left\langle v^{*}, s\right\rangle}{\|s\|}=: v<0$, we have

$$
D_{N}^{*} N(\hat{x}, \hat{y})\left(y^{*}\right)=F(\hat{z})^{*} y^{*}+N(\hat{x}, K)
$$

Proof (i) Since $F$ is Fréchet differentiable at $\hat{z}$, we get $\hat{D}^{*} F(\hat{z})\left(y^{*}(\hat{x}-\hat{z})^{*}\right)=\nabla F(\hat{z})^{*}\left(y^{*}(\hat{x}-\hat{z})^{*}\right)$. The result immediately follows from Theorem 4.2. 
(ii) Since $K$ is regular at $\hat{x}$, we have $\hat{N}(\hat{x}, K)=N(\hat{x}, K)$. Then

$$
\begin{aligned}
D_{M}^{*} N(\hat{x}, \hat{y})\left(y^{*}\right) & \subset F(\hat{z})^{*} y^{*}+N(\hat{x}, K) \\
& =F(\hat{z})^{*} y^{*}+\hat{N}(\hat{x}, K) \\
& =\hat{D}^{*} N(\hat{x}, \hat{y})\left(y^{*}\right) \\
& \subset D_{M}^{*} N(\hat{x}, \hat{y})\left(y^{*}\right) .
\end{aligned}
$$

Thus, we have

$$
D_{M}^{*} N(\hat{x}, \hat{y})\left(y^{*}\right)=\hat{D}^{*} N(\hat{x}, \hat{y})\left(y^{*}\right)=F(\hat{z})^{*} y^{*}+N(\hat{x}, K)
$$

which implies $N$ is $M$-regular at $(\hat{x}, \hat{y})$.

(iii) It can be proved similar to case (ii). This completes the proof.

\section{Competing interests}

The authors declare that they have no competing interests.

\section{Authors' contributions}

All authors contributed equally to the writing of this paper. All authors read and approved the final manuscript.

\section{Author details}

${ }^{1}$ College of Mathematics and Statistics, Nanyang Normal University, Nanyang, Henan 473061, China. ${ }^{2}$ College of Statistics and Mathematics, Yunnan University of Finance and Economics, Kunming, Yunnan 650221, China.

\section{Acknowledgements}

The first author was supported by the Scientific Research Fund for Advanced Talents of Nanyang Normal University. The second author was supported by the National Natural Science Foundation of Yunnan Province (No. 2014FD023). The authors would like to thank the anonymous referees for their valuable comments and suggestions, which helped to improve the paper.

Received: 13 January 2015 Accepted: 5 June 2015 Published online: 17 September 2015

\section{References}

1. Giannessi, F: Theorems of alternative, quadratic programs and complementarity problems. In: Cottle, RW, Giannessi, F, Lions, JL (eds.) Variational Inequalities and Complementarity Problems, pp. 151-186. Wiley, Chichester (1980)

2. Chen, GY: Existence of solutions for a vector variational inequality: an extension of Hartman-Stampacchia theorem. J. Optim. Theory Appl. 74, 445-456 (1992)

3. Lee, GM, Kim, DS, Lee, BS: Vector variational inequality as a tool for studying vector optimization problems. Nonlinear Anal. 34, 745-765 (1998)

4. Cheng, YH, Zhu, DL: Global stability results for the weak vector variational inequality. J. Glob. Optim. 32, 543-550 (2005)

5. Li, SJ, Chen, GY, Teo, KL: On the stability of generalized vector quasi-variational inequality problems. J. Optim. Theory Appl. 113, 283-295 (2002)

6. Daniele, P, Maugeri, A: Vector variational inequalities and modeling of a continuum traffic equilibrium problem. In: Giannessi, F (ed.) Vector Variational Inequality and Vector Equilibria: Mathematical Theories, pp. 97-109. Kluwer Academic, Boston (2000)

7. Goh, CJ, Yang, XQ: Vector equilibrium problem and vector optimization. Eur. J. Oper. Res. 116, 615-628 (1999)

8. Chen, GY, Goh, CJ, Yang, XQ: On gap functions for vector variational inequalities. In: Giannessi, F (ed.) Vector Variational Inequality and Vector Equilibria: Mathematical Theories, pp. 55-70. Kluwer Academic, Boston (2000)

9. Li, SJ, Yan, H, Chen, GY: Differential and sensitivity properties of gap functions for vector variational inequalities. Math. Methods Oper. Res. 57, 377-391 (2003)

10. Meng, KW, Li, SJ: Differential and sensitivity properties of gap functions for Minty vector variational inequalities. J. Math. Anal. Appl. 337, 386-398 (2008)

11. Li, MH, Li, SJ: Second-order differential and sensitivity properties of weak vector variational inequalities. J. Optim. Theory Appl. 144, 76-87 (2010)

12. Mordukhovich, BS: Metric approximations and necessary optimality conditions for general classes of extremal problems. Sov. Math. Dokl. 22, 526-530 (1980)

13. Bao, TQ, Mordukhovich, BS: Relative Pareto minimizers for multiobjective problems: existence and optimality conditions. Math. Program., Ser. A 122, 301-347 (2010)

14. Dutta, J, Tammer, C: Lagrangian conditions for vector optimization in Banach spaces. Math. Methods Oper. Res. 64, 521-541 (2006) 
15. Ye, JJ, Zhu, QJ: Multiobjective optimization problem with variational inequality constraints. Math. Program., Ser. A 96, 139-160 (2003)

16. Levy, AB, Mordukhovich, BS: Coderivatives in parametric optimization. Math. Program., Ser. A 99, 311-327 (2004)

17. Lucet, $Y, Y$, JJ: Sensitivity analysis of the value function for optimization problems with variational inequality constraints. SIAM J. Control Optim. 40, 699-723 (2001); Erratum SIAM J. Control Optim. 41, 1315-1319 (2002)

18. Outrata, JV: Optimality conditions for a class of mathematical programs with equilibrium constraints. Math. Oper. Res. 24, 627-644 (1999)

19. Zhang, R: Weakly upper Lipschitzian multifunctions and applications to parametric optimization. Math. Program. Ser. A 102, 153-166 (2005)

20. Li, SJ, Xue, XW: Sensitivity analysis of gap functions for vector variational inequality via coderivatives. Optimization 63(7), 1075-1098 (2014)

21. Mordukhovich, BS: Variational Analysis and Generalized Differentiation, Vol. I: Basic Theory, Vol. II: Applications Springer, Berlin (2006)

22. Kruger, AY, Mordukhovich, BS: Extremal points and the Euler equation in nonsmooth optimization. Dokl. Akad. Nauk SSSR 24, 684-687 (1980)

23. Mordukhovich, BS: Maximum principle in problems of time optimal control with nonsmooth constraints. J. Appl. Math. Mech. 40, 960-969 (1976)

24. Mordukhovich, BS: Coderivatives of set-valued mappings: calculus and applications. Nonlinear Anal. 30, 3059-3070 (1997)

25. Robinson, SM: Generalized equations and their solutions, I: basic theory. Math. Program. Stud. 10, 128-141 (1979)

26. Tanino, T: Sensitivity analysis in multiobjective optimization. J. Optim. Theory Appl. 56, 479-499 (1988)

27. Chuong, TD: Normal subdifferentials of efficient point multifunctions in parametric vector optimization. Optim. Lett. 7, 1087-1117 (2013)

\section{Submit your manuscript to a SpringerOpen ${ }^{\circ}$ journal and benefit from:}

- Convenient online submission

Rigorous peer review

- Immediate publication on acceptance

- Open access: articles freely available online

- High visibility within the field

- Retaining the copyright to your article 